\title{
Correction to: Assessment of Autism Spectrum Disorder in Deaf Adults with Intellectual Disability: Feasibility and Psychometric Properties of an Adapted Version of the Autism Diagnostic Observation Schedule (ADOS-2)
}

\author{
D. Holzinger ${ }^{1,2,3} \cdot$ C. Weber ${ }^{1,4} \cdot$ S. Bölte $5^{5,6,7} \cdot$ J. Fellinger ${ }^{1,2,8} \cdot$ J. Hofer ${ }^{1,2,9}$ (1)
}

Published online: 17 August 2021

๑) Springer Science+Business Media, LLC, part of Springer Nature 2021

\section{Correction to: \\ Journal of Autism and Developmental Disorders https://doi.org/10.1007/s10803-021-05203-5}

Correction regarding Table 1 and the corresponding text passage under "Participants and Study Setting". Corrections are highlighted bold.
Participants and Study Setting (3rd paragraph)

The sample had a mean age of 44.6 years (range 17 to $75, \mathrm{SD}=18.7)$ and a majority was male $(65 \%)$. The level of intellectual functioning varied. While $50 \%$ had a cognitive level of functioning above an age of reference (AOR) of 6 years, in 50\% the AOR was below 6 years. The degree of hearing loss was....
The original article can be found online at https://doi.org/10.1007/ s10803-021-05203-5.

\section{J. Hofer}

johannes.hofer@bblinz.at

1 Forschungsinstitut für Entwicklungsmedizin, Johannes Kepler Universität Linz, Linz, Austria

2 Institut für Sinnes- und Sprachneurologie, Konventhospital Barmherzige Brüder, Seilerstätte 2, 4021 Linz, Austria

3 Institut für Sprachwissenschaft, Karl-Franzens-Universität Graz, Graz, Austria

4 Institut für Inklusive Pädagogik, Pädagogische Hochschule OÖ, Linz, Austria

5 Department of Women's and Children's Health, Center of Neurodevelopmental Disorders (KIND), Centre for Psychiatry Research, Karolinska Institutet \& Stockholm Health Care Services, Region Stockholm, Stockholm, Sweden

6 Child and Adolescent Psychiatry, Stockholm Health Care Services, Region Stockholm, Stockholm, Sweden

7 Curtin Autism Research Group, Curtin School of Allied Health, Curtin University, Perth, WA, Australia

8 Abteilung für Sozialpsychiatrie der Universitätsklinik für Psychiatrie und Psychotherapie, Medizinische Universität Wien, Vienna, Austria

9 Abteilung für Pädiatrie I, Medizinische Universität Innsbruck, Innsbruck, Austria 
Table 1 Participant characteristics

\begin{tabular}{|c|c|c|c|}
\hline & Modul $1(n=8)$ & Modul $2(n=16)$ & Modul $3(\mathrm{n}=32)$ \\
\hline Age M (SD) & $41.25(19.91)$ & $37.00(18.52)$ & $49.00(17.48)$ \\
\hline Sex male $\mathrm{n}(\%)$ & $4(50.0)$ & $14(87.5)$ & $19(59.4)$ \\
\hline Cognitive functioning $\mathrm{AOR}^{\mathrm{a}} \mathrm{M}$ (SD) & $3.28(1.58)$ & $4.80(1.39)$ & $6.77(1.73)$ \\
\hline \multicolumn{4}{|l|}{ Cognitive functioning $\operatorname{AOR}^{\mathrm{a}} \mathrm{n}(\%)$} \\
\hline$\geq 9$ years & $\mathbf{0}(\mathbf{0 . 0})$ & $1(6.3)$ & $4(12.5)$ \\
\hline$<9$ to 6 years & $1(12.5)$ & $1(6.3)$ & $21(65.6)$ \\
\hline$<6$ to 3 years & $4(50.0)$ & $14(87.5)$ & 7 (21.9) \\
\hline$<3$ years & 3 (37.5) & $\mathbf{0}(\mathbf{0 . 0})$ & $\mathbf{0}(\mathbf{0 . 0})$ \\
\hline \multicolumn{4}{|l|}{ Hearing loss $\mathrm{n}(\%)$} \\
\hline Moderate & $0(0.0)$ & $0(0.0)$ & $1(3.1)$ \\
\hline Severe & $0(0.0)$ & $1(6.3)$ & $5(15.6)$ \\
\hline Profound & $8(100.0)$ & $15(93.8)$ & $26(81.3)$ \\
\hline Cerebral Palsy n (\%) & $4(50.0)$ & $2(14.3)$ & $7(21.9)$ \\
\hline Epilepsy $^{\mathrm{a}} \mathrm{n}(\%)$ & $5(62.5)$ & $6(42.9)$ & $4(12.5)$ \\
\hline Psychiatric Diagnosis n (\%) & $1(12.5)$ & $3(21.4)$ & $10(32.3)$ \\
\hline
\end{tabular}

${ }^{\mathrm{a}}$ significant differences $(\mathrm{p}<0.05)$ between modules (Non-parametric One-way Anova for continuous variables and exact Fisher tests for categorical variables)

Publisher's Note Springer Nature remains neutral with regard to jurisdictional claims in published maps and institutional affiliations. 